\title{
Le renseignement comme objet de recherche en SHS : le rôle central des SIC
}

Information as a research subject in SHS: the central role of SIC

Franck Bulinge et Eric Boutin

\section{(2) OpenEdition}

\section{Journals}

Édition électronique

URL : http://journals.openedition.org/communicationorganisation/4951

DOI : 10.4000/communicationorganisation.4951

ISSN : 1775-3546

Éditeur

Presses universitaires de Bordeaux

Édition imprimée

Date de publication : 1 juin 2015

Pagination : 179-195

ISSN : 1168-5549

Référence électronique

Franck Bulinge et Eric Boutin, «Le renseignement comme objet de recherche en SHS : le rôle central des SIC », Communication et organisation [En ligne], 47 | 2015, mis en ligne le 01 juin 2018, consulté le 30 avril 2019. URL : http://journals.openedition.org/communicationorganisation/4951 ; DOI :

10.4000/communicationorganisation.4951 


\title{
Le renseignement comme objet de recherche en SHS : le rôle central des SIC
}

\author{
Franck Bulinge ${ }^{1}$ et Eric Boutin²
}

\section{Introduction}

En 1881, le colonel Jules Louis Lewal écrit : «la science des renseignements, leur recherche et leur emploi, est la branche la moins connue, la plus négligée jusqu'ici surtout en France. On l'a considérée comme une partie accessoire, à laquelle chacun était naturellement apte et qui n'avait nullement besoin d'être étudiée. » (Lewal 1881). Un peu plus d'un siècle plus tard, CoutauBégarie (1999) précise : " tous les théoriciens s'accordent à reconnaître que le renseignement est indispensable à la décision stratégique. C'est tellement évident qu'il n'est plus nécessaire d'y revenir. La grande majorité des auteurs s'en tiennent à cette pétition de principe. Le renseignement se trouve ainsi réduit à sa portion congrue.»

De fait, alors que les chercheurs anglo-saxons ont publié de nombreux travaux sur le renseignement depuis les années 1950, on ne peut que constater la rareté des travaux français en sciences humaines et sociales (Chopin $2011)^{3}$. Certes, la première thèse consacrée au renseignement est soutenue en France (Colonieu 1888), et sera suivie par quelques autres jusqu'à la Seconde guerre mondiale (Detourbet 1898 ; Routier 1913 ; Cavadia 1926 ; Alteirac 1935 ; Bonte 1936 ; Wolfgang 1937). Toutefois, la recherche connaît une éclipse quasi-totale durant cinquante ans, alors même que les Anglo-Saxons développent dès la fin de la guerre, un courant de recherche universitaire appelé Intelligence Studies. Il faut attendre 1994 pour voir " renaître » les études françaises sur le renseignement, à la faveur d'une tentative de l'amiral Pierre Lacoste pour tendre une passerelle entre le monde fermé du renseignement

\footnotetext{
1 Franck Bulinge est Maître de conférences, docteur habilité à diriger des recherches en sciences de l'information et de la communication, chercheur au laboratoire I3M, Université de Toulon ; bulinge@univ-tln.fr

2 Eric Boutin est Professeur des Universités, docteur en sciences de l'information et de la communication, chercheur au laboratoire I3M, Président de 1'Université de Toulon ; boutin@univ-tln.fr

3 Sur la recension des travaux anglo-saxons, nous invitons le lecteur à lire l'étude de Chopin et al. (2011), Étudier le renseignement. État de l'art et perspectives de recherche, Études de 1'IRSEM, n 9, 2011. Ce document est téléchargeable en ligne.
} 
et celui de la recherche universitaire. Ainsi émergent quelques travaux issus de diverses disciplines des sciences humaines et sociales : anthropologie, histoire, politique, droit, information et communication (Dewerpe 1994 ; Lacoste 1997, 1998 ; Beau 1997 ; Warusfeld 2000 ; Soutou 2001 ; Forcade 2005, 2008 ; Laurent 2005, 2009a, 2009b ; Bulinge 2006, 2010). Mais ils restent anecdotiques et ne parviennent pas à s'imposer dans leurs disciplines respectives.

Au même moment, le renseignement occidental ${ }^{4}$ se trouve au cœur d'une révolution épistémologique que mettent en évidence deux événements singuliers. Le premier est l'appel à contribution émis en 2005 par l'association américaine Mitre suite au constat d'échec des services de renseignement après les attentats du 11 septembre 2001. Le second est l'appel d'offre du ministère de la Défense, en novembre 2010, au profit de la Direction du renseignement militaire (DRM), portant sur les "fondamentaux épistémologiques du renseignement et de l'intelligence économique $»^{5}$. Confirmant l'absence de réflexion académique dans ce domaine et la nécessité de faire émerger une "science du renseignement » basée sur les travaux universitaires, cet appel d'offre fait suite au Livre blanc sur la défense et la sécurité nationale qui met en exergue la nécessité de développer des recherches en sciences humaines et sociales afin de répondre aux enjeux stratégiques du XXI ${ }^{\mathrm{e}}$ siècle (LB 2008).

Ainsi émerge une problématique liée à la nécessaire évolution du " modèle de renseignement occidental », face à l'évolution des relations internationales, elles-mêmes en proie à un débat épistémologique (Rioux et al., 1988 ; Klotz et Lynch 1999 ; Dussouy 2006). L'enjeu s'impose comme une évidence : à défaut de s'adapter à l'évolution du monde, le concept de renseignement est voué à l'obsolescence. Dans cette perspective, la question de recherche qui vient naturellement à l'esprit est la suivante : la recherche universitaire peutelle contribuer à cette évolution?

Le but de cet article est de montrer que le renseignement peut être envisagé comme un objet de recherche pluri et interdisciplinaire en sciences humaines et sociales et que, par ailleurs, et dans la mesure où il est avant tout une activité de traitement d'information, il trouve naturellement sa place dans le champ des sciences de l'information et de la communication (SIC).

Dans une première partie, nous définissons le concept de renseignement et nous mettons en évidence ses multiples dimensions au regard des sciences humaines et sociales. Dans une deuxième partie, nous proposons d'explorer les pistes de recherche qu'offre cet objet dans le champ interdisciplinaire des sciences de l'information et de la communication (SIC).

4 La notion de « modèle de renseignement occidental » renvoie à un concept développé depuis plus d'un siècle au sein des démocraties européennes et aux États-Unis. Il n'existe pas d'étude comparative entre ce modèle et ceux développés dans les pays de l'Est (ex-URSS) ou en Chine. Par prudence, nous utiliserons donc le terme « renseignement occidental » pour décrire un objet explicitement décrit dans la documentation disponible, sans préjuger des modèles russes et chinois.

5 Avis d'appel public à la concurrence n 10352 du 30 novembre 2010. 


\section{Première partie: le concept de renseignement}

Le concept de renseignement est né à la fin du XIX ${ }^{\mathrm{e}}$ siècle. On passe, à cette époque, de la notion triviale d'espionnage, qui correspond à des pratiques clandestines plus ou moins formalisées au service du Prince, à l'institution d'une bureaucratie secrète au service de l'État (Dewerpe 1994 ; Laurent 2009).

Le modèle du renseignement occidental se construit durant le $\mathrm{XX}^{\mathrm{e}}$ siècle sur des bases empiriques, dans le secret des services spéciaux et dans un climat de méfiance entretenu par la crainte d'être soi-même espionné. Parallèlement, la littérature et le cinéma construisent une représentation plus ou moins fantasmée de cet univers du secret. Cela explique en grande partie le paradoxe qui fait du renseignement un objet familier et néanmoins mal connu.

\section{Définition académique}

Il est intéressant, dans un premier temps, de relever les définitions du terme « renseignement » dans trois des principaux dictionnaires scolaires :

Larousse : 1) Indication, information, éclaircissement donnés sur quelqu'un, quelque chose : Donner des renseignements sur une affaire. 2) Activité visant à acquérir et à tenir à jour la connaissance de l'ennemi ou des puissances étrangères.

Petit Robert : 1) Ce par quoi on fait connaître quelque chose à quelqu'un. Avis, indication, information, tuyau. 2) Information concernant l'ennemi, et tout ce qui met en danger l'ordre public, la sécurité. Recherche de telles informations.

Hachette : 1) Une indication fournie sur quelqu'un ou quelque chose. 2) L'ensemble des informations dont le commandement a besoin pour élaborer sa ligne de conduite

Il en ressort que, pour nos académiciens, le terme « renseignement » renvoie principalement à la notion d'information. Seul, le Larousse évoque l'activité visant à acquérir et à tenir à jour ces informations.

Ces définitions sont particulièrement réductrices et ne permettent pas de cerner précisément le concept, entretenant de fait le flou conceptuel évoqué par Dobry.

\section{Le renseignement vu par les praticiens}

Chopin (2011) souligne l'absence de définition théorique précise dans la littérature consacrée au renseignement. Il précise que les définitions existantes renvoient généralement à des concepts techniques. Cela s'explique principalement par le fait que le renseignement est d'abord une pratique empirique avant d'être un objet théorique. Henri (1998) le définit comme une démarche opératoire, confirmant la vision praxéologique qu'en ont les auteurs issus du monde du renseignement depuis plus d'un siècle, et qui s'ancre dans une culture de l'action (Bulinge 2012). 
Cette faiblesse terminologique est à l'origine d'un flou conceptuel qui renvoie opportunément, selon Dobry (1997), à ce que la tradition sociologique appelle les «fonctions sociales floues ».

Dans la littérature, le terme « renseignement » renvoie généralement à trois définitions entrelacées : il est à la fois « une sorte de connaissance, l'organisation qui la produit et l'activité de cette organisation $»^{6}$ (Kent 1966, cité par Chopin 2011). L'entrelacement des trois niveaux de définition est difficile à dénouer, et n'est généralement pas débattu dans le milieu du renseignement. Ainsi, pour le manuel de renseignement des sous-officiers (TTA1507), " un » renseignement est "le résultat de l'exploitation d'informations ", tandis que « le " renseignement est « l'ensemble des activités qui permettent de répondre aux besoins en renseignements (orientation, recherche, exploitation, diffusion) » et « l'ensemble des organismes qui se consacrent à ces activités. »

\section{a) Renseignement, information, connaissance}

Dans cette première acception, le renseignement est entendu compris comme une connaissance de l'ennemi utile au chef (Lewal 1881 ; D'Esclaibes 1944).

Cette connaissance est, selon Lacoste (2002), « une information élaborée, pertinente et utile, correspondant aux besoins de celui qui la reçoit, qui doit donc lui parvenir à bon escient et en temps utile pour qu'il puisse en tirer profit ». Pour Baud (1998), « un renseignement est une information évaluée et exploitée ayant passé le cycle du renseignement et prête à être diffusée à un client ». À travers cette définition, l'auteur fait la distinction entre le renseignement et l'information, considérant cette dernière comme un matériel non évalué et non exploité. Le PIA 02-200 définit l'information comme un " renseignement brut ", alors que le renseignement serait un produit finalisé, c'est-à-dire une connaissance utile au décideur. Krizan reprend la définition du capitaine William S. Brei : «Le renseignement est plus que de l'information. C'est de la connaissance qui a été spécialement préparée pour les circonstances particulières d'un client. $»^{8}$.

Certains auteurs semblent vouloir distinguer information et renseignement en fonction du caractère secret ou public, autrement dit de l'accès à l'information. Ainsi, pour Silberzahn (1995) et Chouet (1997), le renseignement a trait à la recherche d'informations secrètes. Réduisant le renseignement à une information secrète et l'activité de renseignement à l'espionnage, ils soutiennent que le renseignement relèverait exclusivement des services

6 "A kind of knowledge, the type of organization which produces the knowledge, and the activity pursued by the intelligence organization".

7 TTA150, Manuel du sous-officier, édition 2008, CEERAT, EMAT/COFAT.

8 "It is knowledge that has been prepared for a customer's unique circumstances", BREI W. S. (1996), Getting Intelligence Right: The Power of Logical Procedure, Occasional paper n 2, Joint Military College, January 1996. 
spéciaux dont l'objectif est de "connaître ce quion veut leur cacher ». Ainsi le terme " renseignement ouvert » serait un oxymore, car ce qu'on appelle abusivement « renseignement ouvert » serait tout simplement de l'information.

Le PIA 02-200 introduit par ailleurs la notion de donnée, définie comme un " élément de connaissance élémentaire (heure, lieu, événement, acteur, moyen, etc.) »'. Dès lors, pour le TTA150, l'information est une

une donnée non traitée, de toute nature, qui peut être utilisée pour l'élaboration du renseignement. De manière plus précise, c'est un élément de connaissance par la signification que l'on tire d'un fait ou d'une donnée. Enfin, une information doit par essence être communiquée. L'information est générée directement par l'homme ou peut être tirée de données fournies par des moyens techniques.

Il s'établit ainsi un lien hiérarchique entre données, informations et renseignement. Les données permettent de produire des informations, lesquelles permettent de produire le renseignement. Il s'ensuit une notion de processus qui vise la transformation des données en informations et des informations en renseignement.

\section{b) Le processus de renseignement}

Le PIA 02-200 définit le renseignement comme le « résultat d'un processus d'exploitation de données et d'informations dont la collecte a été orientée et décidée pour répondre à un besoin décisionnel ». Le document précise qu'il s'agit d'" une information validée, ou une évaluation tirée d'informations validées, portant sur une "cible" non coopérante et destinée spécifiquement à la satisfaction du besoin d'un décideur ou d'un autre utilisateur, de quelque niveau qu'il soit ». Le PIA 02-200 rejoint ainsi l'idée généralement partagée (Baud 1998 ; Krizan 1999 ; Lacoste 2002 ; Clark 2007) selon laquelle le renseignement est le résultat d'un processus d'exploitation de données et d'informations dont la collecte a été orientée et décidée pour répondre à un besoin décisionnel.

Ce processus se décompose en une série d'étapes distinctes : expression des besoins en renseignements, recherche/collecte des données, évaluation/ traitement des données, analyse/synthèse des informations et diffusion des renseignements sous forme de synthèses. Il est illustré par la figure suivante :

\section{c) La notion de système organisé: Le cycle du renseignement}

La notion de processus et la dimension téléologique du renseignement introduisent l'idée d'une connaissance produite par un dispositif sociotechnique au sein d'une organisation. Dès lors, il manque au schéma précédent l'idée d'une dynamique sociale de production et notamment le principe d'interaction

9 TTA150 édition 2008, CEERAT, EMAT/COFAT. 


\section{$C \& O n^{\circ} 47$}

systémique qui en découle. On introduit par conséquent la notion de cycle du renseignement qui illustre cette dynamique communicante dans un processus continu centré sur la satisfaction d'un besoin de connaissance exprimé par le décideur. Le cycle du renseignement donne tout à la fois un aperçu de la fonction, de l'organisation, de la structure et du processus théoriques de pilotage et de production du renseignement.

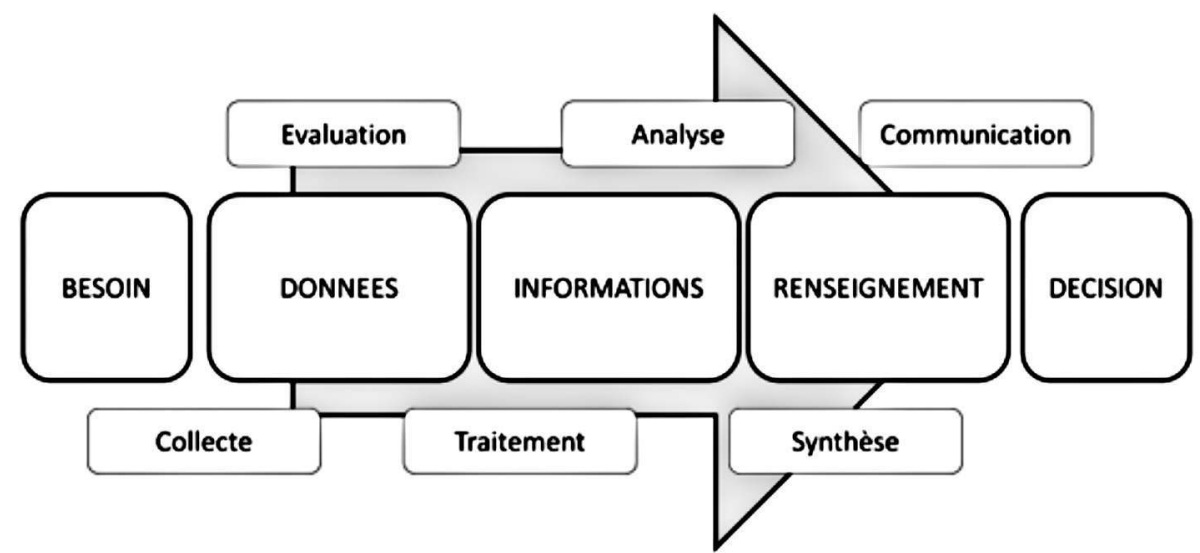

Figure 1 : Processus de renseignement (d'après Bulinge, 2012)

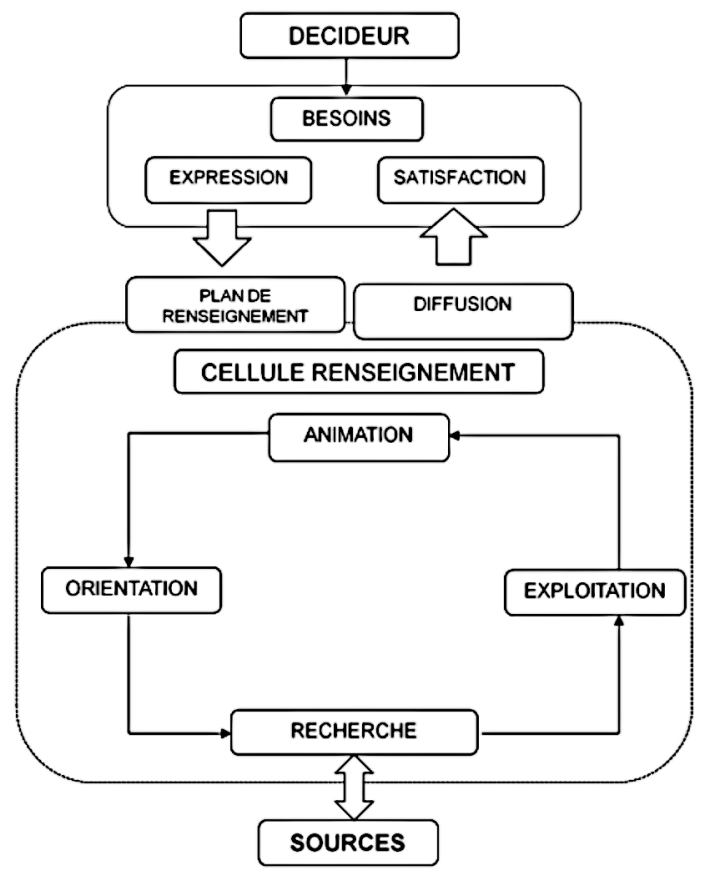

Figure 2 : Le cycle du renseignement (d'après Faivre, 1991) 


\section{d) L'activité de renseignement:}

Suivant la logique de processus décrite plus haut, le renseignement se décompose en deux activités :

- Acquisition des données : en fonction des besoins exprimés par le décideur, cette activité consiste à organiser la recherche de données en fonction des ressources disponibles. Les ressources sont ici comprises au sens de « capteurs » et d' « agents ». Les capteurs sont des dispositifs technologiques de recueil de données (imagerie, interceptions radioélectriques, etc.), tandis que les agents sont des sources humaines d'informations.

- Exploitation : cette activité se décompose en trois phases (traitement/ computation, analyse, rédaction de synthèses) et vise la transformation des données en informations puis en renseignement, lequel est transmis au décideur.

En résumé, le renseignement est un objet complexe dont la définition actuelle repose sur l'expérience des acteurs, selon une logique praxéologique. L'absence de recherche académique est cependant perceptible. Il manque, en effet, une exploration systématique des multiples facettes de cet objet, comme le montrent les premiers travaux de recherche menés sur l'anthropologie (Dewerpe, 1994), l'épistémologie du renseignement (Ben Israël 2004), et la critique du cycle du renseignement, emblématique d'une méthodologie « mécaniste» du renseignement (Clark 2004 ; Bulinge 2006). Ces quelques travaux scientifiques ont mis en évidence les failles d'un modèle séculaire que Bulinge (2012) qualifie de « science proverbiale » parce que fondée sur des aphorismes, tel celui d'Auguste Comte auquel ont recours les écoles de renseignement : "savoir pour préroir afin de pouvoir ».

\section{Deuxième partie : les champs disciplinaires du renseignement}

\section{Domaines et acteurs du renseignement}

Destiné à produire des connaissances utiles aux dirigeants politiques et militaires, le renseignement touche à de multiples domaines. On parle de renseignement politique, militaire, économique, technologique, etc. Le « renseignement extérieur » a trait aux activités de politique étrangère, tandis que le « renseignement intérieur » désigne les activités de sécurité intérieure. S'inscrivant dans un processus décisionnel, le renseignement peut se situer au niveau stratégique, opérationnel ou tactique. S'agissant des sources, on parle de renseignement humain (agents) ou de renseignement technique (capteurs), lequel se subdivise en renseignement électromagnétique (interception radiotéléphoniques), image (satellites). Le renseignement ouvert désigne l'activité centrée sur la collecte et l'exploitation d'informations publiques (veille stratégique). Le cyber-renseignement désigne l'activité de pénétration illicite des réseaux numériques. 


\section{$C \& O n^{\circ} 47$}

Les acteurs du renseignement sont regroupés au sein d'une communauté à la fois contrainte et protégée par une organisation et des règles liées à la nature confidentielle de leurs activités. Il existe une grande diversité de métiers au sein d'un service de renseignement, suggérant des connaissances théoriques et pratiques qui relèvent aussi bien des sciences dures que des sciences humaines et sociales. Les plus emblématiques sont directement liés aux activités décrites précédemment : linguistes et traducteurs, officiers traitants (manipulation des sources humaines), cryptanalystes (activité de cryptologie), interprétateurs d'images, analystes géopolitiques, informaticiens, etc.

Au regard de ces métiers, les disciplines les plus sollicitées apparaissent en filigrane : histoire, géographie, science politique, sociologie, anthropologie, psychologie, cognition, linguistique, sémiotique, statistiques, sciences de l'information.

Cette énumération intuitive peut être corroborée par une analyse plus précise des contributions disciplinaires au regard des différentes étapes du cycle du renseignement, comme l'indique le tableau suivant :

\begin{tabular}{|c|c|c|c|c|c|c|c|}
\hline & Orientation & Recherche & & Traitement/ & ploitation & & Diffusion \\
\hline & & & Traduction & Evaluation & Analyse & Synthèse & \\
\hline Anthropologie & & $\mathrm{X}$ & & $\mathrm{X}$ & $\mathrm{X}$ & $\mathrm{X}$ & $\mathrm{X}$ \\
\hline Cognition & $\mathrm{X}$ & $\mathrm{X}$ & $\mathrm{X}$ & $\mathrm{X}$ & $\mathrm{X}$ & $\mathrm{X}$ & $\mathrm{X}$ \\
\hline Droit & $\mathrm{X}$ & $\mathrm{X}$ & & & & & $\mathrm{X}$ \\
\hline Economie & $\mathrm{X}$ & & & & $\mathrm{X}$ & & \\
\hline Epistémologie & & $\mathrm{X}$ & & $\mathrm{X}$ & $\mathrm{X}$ & $\mathrm{X}$ & $\mathrm{X}$ \\
\hline Géographie & $\mathrm{X}$ & $\mathrm{X}$ & & & $\mathrm{X}$ & & \\
\hline Histoire & $\mathrm{X}$ & & & $\mathrm{X}$ & $\mathrm{X}$ & & \\
\hline SIC & $\mathrm{X}$ & $\mathrm{X}$ & $\mathrm{X}$ & $\mathrm{X}$ & $\mathrm{X}$ & $\mathrm{X}$ & $\mathrm{X}$ \\
\hline Linguistique & $\mathrm{X}$ & $\mathrm{X}$ & $\mathrm{X}$ & & $\mathrm{X}$ & $\mathrm{X}$ & $\mathrm{X}$ \\
\hline Management & $\mathrm{X}$ & $\mathrm{X}$ & & & $\mathrm{X}$ & & \\
\hline Politique & $\mathrm{X}$ & & & & $\mathrm{X}$ & $\mathrm{X}$ & $\mathrm{X}$ \\
\hline Psychologie & $\mathrm{X}$ & $\mathrm{X}$ & & $\mathrm{X}$ & $\mathrm{X}$ & $\mathrm{X}$ & $\mathrm{X}$ \\
\hline Religion & & $\mathrm{X}$ & $\mathrm{X}$ & $\mathrm{X}$ & $\mathrm{X}$ & & \\
\hline Sémiotique & & & & & $\mathrm{X}$ & $\mathrm{X}$ & $\mathrm{X}$ \\
\hline
\end{tabular}




\begin{tabular}{|l|c|c|c|c|c|c|c|}
\hline & Orientation & Recherche & \multicolumn{4}{|c|}{ Traitement/Exploitation } & Diffusion \\
\hline & & & Traduction & Evaluation & Analyse & Synthèse & \\
\hline Sociologie & $\mathrm{X}$ & & & & $\mathrm{X}$ & $\mathrm{X}$ & $\mathrm{X}$ \\
\hline Statistiques & & & & $\mathrm{X}$ & $\mathrm{X}$ & & \\
\hline
\end{tabular}

Tableau 1 : Principales disciplines sollicitées par le cycle du renseignement

Cette approche est intéressante en ce qu'elle identifie les savoirs nécessaires à l'exercice des métiers cités plus haut, dans le cadre de la mise en œuvre du cycle du renseignement. Autrement dit, c'est ce que l'université peut apporter en termes de formation aux élèves et aux personnels des services de renseignement.

De la même manière, il est intéressant d'identifier les disciplines scientifiques susceptibles de contribuer à l'évolution théorique et méthodologique du modèle de renseignement. C'est ce que nous proposons à travers l'analyse des dimensions du renseignement.

\section{Les dimensions pluridisciplinaires du renseignement}

En tant qu'objet complexe, le renseignement s'inscrit dans de multiples dimensions qui renvoient aux diverses disciplines des sciences humaines et sociales, comme le montre le tableau suivant (d'après Bulinge 2012) :

\begin{tabular}{|l|l|l|}
\hline Dimensions & \multicolumn{1}{|c|}{ Problématiques } & Disciplines concernées \\
\hline Théorique & $\begin{array}{l}\text { Ensemble des savoirs, bases épistémologiques et } \\
\text { méthodologiques. Pose la question du renseignement } \\
\text { en tant que processus de construction de } \\
\text { connaissances. }\end{array}$ & $\begin{array}{l}\text { Epistémologie, } \\
\text { philosophie, Infocom, } \\
\text { droit, politique, histoire }\end{array}$ \\
\hline Historique & $\begin{array}{l}\text { Pose la question des origines et de l'évolution. } \\
\text { Elle met en lumière la continuité et les ruptures } \\
\text { paradigmatiques du renseignement. }\end{array}$ & Histoire, politique, droit \\
\hline Téologique & $\begin{array}{l}\text { Relative aux finalités du renseignement. Elle pose } \\
\text { ainsi la question de l'utilité et de la pertinence du } \\
\text { renseignement au regard de la politique et de la } \\
\text { stratégie. }\end{array}$ & $\begin{array}{l}\text { Histoire, politique, } \\
\text { sociologie }\end{array}$ \\
\hline Technologique & $\begin{array}{l}\text { Caractérise l'environnement technologique du } \\
\text { renseignement, et notamment les moyens mis en } \\
\text { œuvre ainsi que la R\&D associée. Pose la question } \\
\text { des moyens. }\end{array}$ & Infocom, anthropologie \\
\hline
\end{tabular}




\section{$C \& O n^{\circ} 47$}

\begin{tabular}{|l|l|l|}
\hline Dimensions & \multicolumn{1}{|c|}{ Problématiques } & Disciplines concernées \\
\hline Economique & $\begin{array}{l}\text { Quelle que puisse être l'importance des autres } \\
\text { dimensions, la dimension économique est } \\
\text { indéniablement celle qui conditionne et traduit } \\
\text { le plus concrètement le niveau des autres. Elle } \\
\text { pose également la question de la rentabilité du } \\
\text { renseignement. }\end{array}$ & $\begin{array}{l}\text { Économie, gestion, } \\
\text { politique }\end{array}$ \\
\hline Sociologique & $\begin{array}{l}\text { Concerne l'ensemble des problématiques } \\
\text { sociales, sociétales, culturelles, éthiques liées au } \\
\text { renseignement. }\end{array}$ & $\begin{array}{l}\text { Sociologie, politique, } \\
\text { infocom, civilisations, } \\
\text { éducation, langage, }\end{array}$ \\
\hline Politique & $\begin{array}{l}\text { Ensemble des problématiques liée aux relations entre } \\
\text { le pouvoir et le renseignement, à son administration } \\
\text { ainsi quà l'impact du renseignement dans l'action } \\
\text { politique et ses conséquences sur le plan de la } \\
\text { sécurité et de la défense nationale. }\end{array}$ & $\begin{array}{l}\text { Politique, sociologie, } \\
\text { histoire }\end{array}$ \\
\hline Juridique & $\begin{array}{l}\text { La dimension juridique renvoie au cadre légal de } \\
\text { son emploi, ainsi qu'aux règles pénales relatives à la } \\
\text { protection des intérêts, à la défense et à la sécurité } \\
\text { nationale. }\end{array}$ & $\begin{array}{l}\text { Droit, philosophie, } \\
\text { sociologie }\end{array}$ \\
\hline Géopolitique & $\begin{array}{l}\text { Questionne le rôle du renseignement dans les } \\
\text { relations internationales }\end{array}$ & $\begin{array}{l}\text { Politique, géographie, } \\
\text { histoire, économie }\end{array}$ \\
\hline
\end{tabular}

Tableau 2: Les multiples dimensions du renseignement

Ce tableau met en évidence non seulement le caractère pluridisciplinaire du renseignement, mais également son caractère interdisciplinaire.

\section{Études vs recherche appliquée}

De fait, il existe deux approches possibles de la recherche ayant pour objet le renseignement. La première est l'approche dite sur le renseignement qui s'inscrit dans une logique pluridisciplinaire de connaissance de l'objet en tant que phénomène. Il s'agit de l'approche que les Anglo-saxons qualifient d'Intelligence Studies et qui consiste pour l'essentiel à étudier le renseignement dans ses dimensions historiques, politiques et culturelles, mais également, quand cela est possible, dans ses dimensions anthropologique (Dewerpe 1994) et praxéologique. La seconde est l'approche dite pour le renseignement. Interdisciplinaire, elle vise l'enrichissement théorique et méthodologique de l'objet en tant que dispositif sociotechnique, soit par l'emprunt aux disciplines des méthodes qui leur sont propres, soit par l'apport de leurs travaux à l'amélioration des modèles existants voire à la conception de nouveaux modèles.

Bien que leurs finalités soient différentes, les deux approches sur et pour le renseignement n'en restent pas moins complémentaires au sens où elles 
s'apportent mutuellement des éléments susceptibles d'enrichir l'objet. Par exemple, les historiens peuvent mettre en évidence le socle culturel sur lequel s'organise la sociologie du renseignement. Associée à cette recherche, une étude anthropologique des pratiques d'analyse permettra de mieux cerner les influences organisationnelles dans la construction du renseignement, ce qui pourrait être utile aux SIC pour la conception d'outils d'aide à l'analyse (Jonhston 2005).

\section{Les SIC : une interdiscipline centrale}

On a tendance à situer naturellement le renseignement dans le champ des sciences politiques en raison de sa vocation à servir les dirigeants politiques. Toutefois, l'objet " renseignement ", envisagé comme instrument politique, apparaît comme une "boîte noire " dont on considère le rôle et l'usage, sans poser la question des processus de construction de connaissances sousjacents. Au cour de cette boîte noire, symbolisée dans la figure $\mathrm{n}^{\circ} 2$ par la cellule de renseignement, se trouve justement le processus de construction du renseignement.

Le tableau $\mathrm{n}^{\circ} 1$ met en évidence l'apport des différentes disciplines à chaque étape du processus, et notamment celui des SIC qui semblent devoir jouer un rôle majeur. À cela rien d'étonnant, si l'on considère, d'une part, que le renseignement a pour vocation de donner du sens à l'environnement stratégique, et, d'autre part, que pour Couzinet (2005) « faire émerger du sens est étroitement dépendant du processus de construction de l'information, du contexte de sa récupération, de l'histoire personnelle de celui qui intervient sur elle, de ses habiletés documentaires, des connaissances qu'il est capable de mobiliser, du contexte dans lequel il se trouve, etc. ». Cela nous amène naturellement à Le Coadic (2006) pour qui la science de l'information a pour objet « l'étude des propriétés générales de l'information (nature, genèse, effets) et l'analyse de ces processus de construction, de communication et d'usages ». Cette définition est bien entendu corroborée par les textes de la section CNU71 qui définit ainsi le champ des SIC :

A. Les études sur les notions d'information et de communication, sur leurs relations, sur la nature des phénomènes et des pratiques ainsi désignés, de même que les différentes approches scientifiques qui s'y appliquent.

B. L'étude, d'une part, des processus, des productions et des usages de l'information et de la communication, d'autre part, de la conception et de la réception de celles-ci. Ainsi que l'étude des processus de médiation et de médiatisation.

C. L'étude des acteurs, individuels et institutionnels, de l'information et de la communication, l'étude des professionnels (dont notamment les journalistes) et de leurs pratiques. 
D. L'étude de l'information, de son contenu, de ses systèmes sous l'angle des représentations, des significations ou des pratiques associées. ${ }^{10}$

De ce point de vue, le renseignement peut être considéré comme un dispositif sociotechnique orienté sur les notions d'information et de communication, s'appuyant sur un processus, produisant et échangeant un type d'information spécifique à un milieu (politique, militaire), à un contexte (relations internationales, guerres), à des acteurs (officiers de renseignement, agents), à des médias (capteurs, systèmes d'information). L'étude du renseignement porte naturellement sur la construction de cette information, sa communication et ses usages. Les SIC sont ainsi amenées à jouer un rôle central dans la recherche appliquée au renseignement. Si l'on considère par exemple que la base du travail de renseignement consiste à collecter, indexer et stocker les informations, on comprend immédiatement l'apport potentiel des recherches sur les méthodes et techniques documentaires. Dans le domaine de l'exploitation de l'information, les SIC, combinées à la psychologie cognitive, permettent d'envisager une critique épistémologique des méthodes existantes, mais également le développement de méthodes prenant en compte les nouvelles approches communicationnelles (Mucchielli 2006, Wolton 2009). On ne peut manquer de faire le lien avec l'intelligence économique qui a trouvé dans les SIC ses principales bases méthodologiques à travers les travaux de différentes équipes (Dou, Rostaing, Manina à Marseille ; Paoli et Lacoste à Marne-la-Vallée, Doucet à Toulouse; Moinet et Marcon à Poitiers ; Dumas, Boutin, Bertacchini, Bulinge à Toulon; David à Nancy). Or, comme l'a montré Bulinge (2002), l'intelligence économique est essentiellement un avatar du renseignement. Justifier l'intelligence économique comme objet de recherche en SIC (Couzinet 2005) revient, par conséquent, à accueillir également le renseignement dans cette discipline.

Il ne s'agit toutefois pas d'enfermer le renseignement dans un carcan disciplinaire. Bien au contraire, dans la mesure où il ne peut être pleinement compris que dans une approche multidimensionnelle. Pour Le Coadic (2006), " la science de l'information est une de ces nouvelles interdisciplines (...) qui voit collaborer entre elles, de manière principale, les disciplines psychologie, linguistique, sociologie, informatique, mathématique, logique, statistique, électronique, économie, droit, philosophie, politique, télécommunications. » Fleury et Walter (2007) posent la question : «Les SIC ne sont-elles pas un espace privilégié où se manifeste une tendance forte et productive heuristiquement dans les SHS contemporaines, soit l'hybridation qui déjoue les découpages hérités d'une approche strucuralo-fonctionnaliste des disciplines ? ». Pour la section CNU71, il n'y a pas de doute possible, " le champ de la section est résolument interdisciplinaire ». Mucchielli (1998) confirme cette vision 
et identifie les différentes disciplines avec lesquelles les SIC partagent des champs de recherche (fig. $n^{\circ} 3$ ).

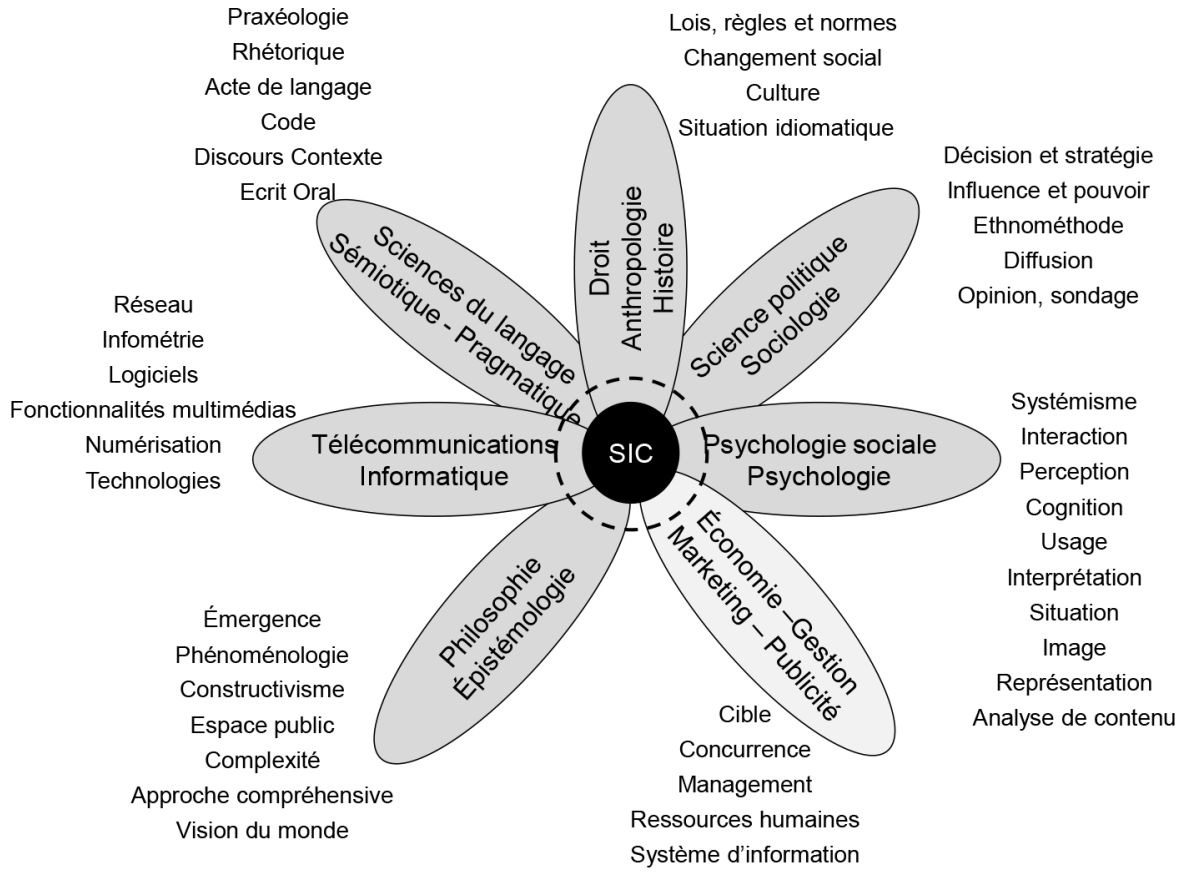

Figure 3: Principaux concepts échangés avec les SIC par les autres disciplines

La grande majorité des items de ce schéma concernent l'activité de renseignement. On peut ainsi soutenir que les SIC constituent un carrefour interdisciplinaire de la recherche sur le renseignement.

Au demeurant, il n'y a là rien d'étonnant et ce n'est qu'un juste retour des choses si l'on considère que les services de renseignement furent les premiers prescripteurs et « usagers » de l'informatique et des techniques documentaires dans lesquelles s'enracine l'histoire des sciences de l'information.

\section{Un exemple de recherche appliquée aux États-Unis}

Voyons à présent un exemple de recherche appliquée pluridisciplinaire. I1 s'agit d'une initiative de l'Office of the Assistant Director of Central Intelligence for Analysis and Production faisant suite au constat d'échec du renseignement américain face aux attentats terroristes du 11 septembre 2001 (Kean et Hamilton 2005). Un colloque international est organisé en mai 2005 par l'association gouvernementale MITRE ${ }^{11}$, fondée en 1958 afin de favoriser 
la recherche dans le domaine de la défense. L'objectif est de faire état des avancées fondamentales, nouvelles et opérationnelles dans le domaine du renseignement ${ }^{12}$.

L'appel à contribution permet de recevoir environ 400 articles et posters, dont 166 ne sont pas classifiés. L'analyse des contributions met en lumière la richesse des problématiques dont beaucoup relèvent des sciences humaines : pensée critique, construction de sens, langage étranger, visualisation d'information, analyse collaborative, supports de décision et simulation, réseaux sociaux, etc. L'analyse des titres et résumés permet de dégager 510 mots-clés dont les principaux sont présentés dans le tableau suivant :

\begin{tabular}{|c|c|c|c|c|}
\hline Étapes du cycle & Mots-clés & Freq & $\%$ & Total \\
\hline \multirow{5}{*}{ Recherche } & Extraction d'information & 23 & 4,51 & \multirow{5}{*}{14,71} \\
\hline & Indexation & 21 & 4,12 & \\
\hline & Sources ouvertes & 15 & 2,94 & \\
\hline & Renseignement humain & 10 & 1,96 & \\
\hline & Traitement du langage étranger & 6 & 1,18 & \\
\hline \multirow{9}{*}{ Exploitation } & Hypothèses concurrentes & 47 & 9,22 & \multirow{9}{*}{31,96} \\
\hline & Fusion du renseignement multisource & 23 & 4,51 & \\
\hline & Renseignement extrait des données massives & 21 & 4,12 & \\
\hline & Analyse multisource & 19 & 3,73 & \\
\hline & Analyse relationnelle & 19 & 3,73 & \\
\hline & \begin{tabular}{|l|} 
Analyse prédictive \\
\end{tabular} & 14 & 2,75 & \\
\hline & Analyse sociale et culturelle & 8 & 1,57 & \\
\hline & Analyse chronologique & 6 & 1,18 & \\
\hline & Formation des analystes & 6 & 1,18 & \\
\hline \multirow{4}{*}{ Diffusion } & Partage, gestion et diffusion des connaissances & 24 & 4,71 & \multirow{3}{*}{8,63} \\
\hline & Argumentation structurée & 10 & 1,96 & \\
\hline & Convivialité & 10 & 1,96 & \\
\hline & TOTAL fréquence/510 & 282 & 55,29 & \\
\hline
\end{tabular}

Tableau 3: Analyse des mots-clés MITRE 2005

Il met en évidence des problématiques d'ordre méthodologique (55\% des contributions retenues). On note ainsi qu'au regard du cycle du renseignement, quelque $32 \%$ des articles concernent l'exploitation, presque $15 \%$ la recherche et le traitement d'information, et enfin 8,6\% les problématiques de communication.

\section{Conclusion}

Le renseignement est un objet encore peu abordé par la communauté universitaire française. Actuellement, il repose sur un socle empirique séculaire qui ne résiste pas à la critique académique. La réforme du renseignement entreprise en 2008, mais également le questionnement épistémologique de la

12 On trouve encore une trace du programme sur http://cryptome.org/intel-2005.htm 
Direction du renseignement militaire, ont clairement soulevé la question de faiblesse de ses fondements épistémologiques.

Envisagé comme un objet de recherche en SHS, le renseignement ouvre un panorama très riche en perspectives compte tenu de son caractère multidimensionnel.

Objet par essence pluridisciplinaire, il appelle dans un premier temps chaque discipline à travailler séparément dans une optique longitudinale. Toutefois, ce type de recherche produisant une « connaissance déconcertée » de caractère inégal, il importe d'envisager la recherche interdisciplinaire susceptible de contribuer non seulement à une meilleure connaissance intrinsèque de l'objet, mais également de contribuer à son évolution. En ce sens, si les études sur le renseignement peuvent être abordées dans toutes les disciplines des SHS, la recherche appliquée revient naturellement à l'interdiscipline que constituent les SIC.

\section{BIBLIOGRAPHIE}

BEAU F. (1996), Renseignement et société de l'information, Fondation pour les études de défense nationale, Librairie de la Documentation française.

BREI W. S. (1996), Getting Intelligence Right: The Power of Logical Procedure, Occasional paper n 2, Joint Military College, January 1996.

BULINGE F. (2006), Le cycle du renseignement : analyse critique d'un modèle empirique, Marketing \& Communication, Éditions Eska, Vol. 3 n 3, octobre 2006, pp. 36-52.

BULINGE F. (2010), Renseignement militaire : une approche épistémologique, Revue internationale d'intelligence informationnelle, Lavoisier, vol. 2-2/2010, juillet-décembre 2010, p. 211-232.

BULINGE F. (2012), De l'espionnage au renseignement, Vuibert-INHESJ.

CHOPIN O. (dir.) (2011), Étudier le renseignement. État de l'art et perspectives de recherche, Études de l'IRSEM, n ${ }^{\circ}$ 9, 2011.

CLARK R. M. (2004), Intelligence Analysis, a Target Centric Approach, CQ Press, Washington DC.

COUTAU-BEGARIE H. (1999), Le renseignement dans la pensée militaire française, Stratégique n 73, 1999/1.

COUZINET V. (2005), Intelligence économique et sciences de l'information et de la communication : quelles questions de recherche? in DAVID A., (dir.) (2005), Organisation des connaissances dans les systèmes d'informations orientés utilisation, contexte de veille et d'intelligence économique, Presses universitaires de Nancy.

DEWERPE A. (1994), Espion, Une anthropologie historique du secret d'état contemporain, Gallimard, Bibliothèque des histoires.

DOBRY M. (1997), « Le renseignement politique dans les démocraties occidentales. Quelques pistes pour l'identification d'un objet flou », Cabiers de la sécurité intérieure (IHESI), n 30, 1997. 
DUSSOUY G. (2006), Traité des relations internationales. Tome 1, Les théories géopolitiques, L'Harmattan.

FERRO C. (2012), L'image des services de renseignement de sécurité : France, RoyaumeUni, Allemagne, Belgique, thèse de doctorat sous la direction de Jacques Barrat, Université de Panthéon-Assas.

FLEURY B., WALTER J. (2007), L'histoire des sciences de l'information et de la communication, Questions de communication, 12/2007, p. 133-148.

FORCADE O. (2008), La république secrète, Histoire des services spéciaux français de 1918 à 1939, Éditions Nouveau Monde.

FORCADE O., LAURENT S. (2005), Secrets d'État, Pouvoir et renseignement dans le monde contemporain, Armand Colin.

HENRI B. (1998), Le renseignement, un enjeu de pouvoir, Economica.

JOHNSTON B. (2005), Analytic Culture in the U.S. Intelligence Community, Center for the Study of Intelligence, Central Intelligence Agency, Washington, DC.

KEAN T. H., HAMILTON L. H. (2005), 11 septembre 2001, Rapport final de la commission nationale sur les attaques terroristes contre les États-Unis, Alban éditions.

KENT S. (1966), Strategic Intelligence for American World Policy, Princeton University Press.

KLOTZ A., LYNCH C. (1999), Le constructivisme dans la théorie des relations internationales, Critique internationale, n' 2, hiver 1999.

KRIZAN L. (1999), Intelligence essential for everyone, occasional paper, Joint Military Intelligence College.

LACOSTE P. (1997), Approches françaises du renseignement, Fondation pour les études de défense, Paris, 1997.

LACOSTE P. (dir.) (1998), Le renseignement à la française, Economica.

LAURENT S. (2009), Politiques de l'ombre, État, renseignement et surveillance de la France, Fayard.

LAURENT S. (dir.) (2009), Politiques du renseignement, Presses universitaires de Bordeaux.

LE COADIC Y.F. (2006), La science de l'information, Que-sais ? PUF.

LEWAL J. (1881), Études de guerre: Tactique des renseignements, Paris, Baudoin, 1881, 2 volumes, réédité en 2010 aux éditions Bibliobazaar.

MATTELART A. (2006), Société de la connaissance, société de l'information, société de contrôle, Cultures Ė Conflits n 64 Hiver 2006 (4/2006) pp. 167-183.

MINDEF (2010), Cabier des clauses particulières n 10352 du 11 octobre 2010, marché relatif aux fondamentaux épistémologiques du renseignement et de l'intelligence économique, Ministère de la défense, secrétariat général de l'administration.

MUCCHIELLI A. (1998), Les sciences de l'information et de la communication.

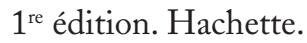

MUCCHIELLI A. (2006), Les sciences de l'information et de la communication. 4 édition. Hachette.

PETER R. (1986), Information is not Intelligence, Army n 36, janvier 1986, p. 34-37. 
RIOUX J-F., KEENES E., LEGARE G. (1988), Le néo-réalisme ou la formulation du paradigme hégémonique en relations internationales, Études internationales, vol. 19, $\mathrm{n}^{\circ} 1,1988$, p. 57-80.

SOUTOU G. H. (dir .) (2001), L'exploitation du renseignement en Europe et aux États-Unis, des années 1930 aux années 1960 : actes du colloque international tenu aux Écoles militaires de Saint-Cyr Coëtquidan, organisé par le Centre de recherche des écoles de Saint-Cyr Coëtquidan, le Centre d'histoire de l'Europe et des relations internationales au $\mathrm{XX}^{\mathrm{e}}$ siècle et le Centre de l'Islam contemporain, Paris IV-Sorbonne, les 3 et 4 juin 1998 .

TREVERTON G. F., JONES S. G., BORAZ S., LIPSCY P., (2006) Toward a Theory of Intelligence : Workshop report (June, 15th, 2005), RAND National Security Research Division, ODNI, 2006.

WOLTON D. (2009), Informer n'est pas communiquer, CNRS Éditions, collection Débats.

Résumé : Longtemps relégué dans le secret du pouvoir politique et militaire, le renseignement a surgi dans le champ scientifique à travers les travaux sur la veille et l'information documentaire (Dou et al., 1990), la bibliométrie (Paoli et al., 1992) et finalement son avatar, l'intelligence économique (Martre, 1994). Face au changement de paradigme que constitue la fin de la guerre froide et la démocratisation des technologies de l'information et de la communication, il devient possible aujourd'hui de faire du renseignement un objet de recherche en sciences humaines et sociales. Le but de cet article est d'introduire le renseignement comme un objet de recherche pluridisciplinaire en sciences humaines et sociales et, dans la mesure où il est avant tout une activité de traitement d'information, de montrer qu'il trouve une place naturelle dans le champ des sciences de l'information et de la communication (SIC).

Mots-clés : renseignement, recherche en SHS, SIC.

Abstract : For a long time, Intelligence has been relegated to the secret of political and military power. But during the 90s, it emerged indirectly in the scientific field through work on documentary information (Dou et al., 1990), bibliometrics (Paoli et al., 1992) and competitive intelligence (Marten, 1994). The aim of this article is to introduce intelligence as a multidisciplinary research topic in humanities and social sciences and, insofar intelligence is primarily an information processing activity, to show that it takes naturally place into the field of information and communication science (SIC).

Keywords : Intelligence studies, Humanities, Information Science. 
